\title{
MÉTODOS MATEMÁTICOS PARA CORREÇÃO DE INTERFERÊNCIAS ESPECTRAIS E EFEITOS INTERELEMENTOS NA ANÁLISE QUANTITATIVA POR FLUORESCÊNCIA DE RAIOS-X
}

\author{
Noemi Nagata, Maria Izabel M. S. Bueno \\ Instituto de Química, Universidade Estadual de Campinas (UNICAMP), CP 6154, 13083-970 Campinas - SP \\ Patricio G. Peralta-Zamora \\ Departamento de Química, Universidade Federal do Paraná (UFPR), CP 19081, 81531-990 Curitiba - PR
}

Recebido em 5/4/00; aceito em 16/11/00

\begin{abstract}
MATHEMATICAL METHODS TO CORRECT SPECTRAL INTERFERENCES AND INTERELEMENTAL EFFECTS IN X-RAY FLUORESCENCE QUANTITATIVE ANALYSIS. One of the main problems in quantitative analysis of complex samples by $\mathrm{x}$-ray fluorescence is related to interelemental (or matrix) effects. These effects appear as a result of interactions among sample elements, affecting the x-ray emission intensity in a non-linear manner. Basically, two main effects occur; intensity absorption and enhancement. The combination of these effects can lead to serious problems. Many studies have been carried out proposing mathematical methods to correct for these effects. Basic concepts and the main correction methods are discussed here.
\end{abstract}

Keywords: x-ray fluorescence; interferences; correction methods.

\section{INTRODUÇÃO}

Nos últimos anos, o conhecimento tem adquirido grandes dimensões e a ciência tem se tornado uma atividade eminentemente multidisciplinar, fundamentada no trabalho conjunto de pesquisadores com elevado grau de especialização. Nestes grupos multidisciplinares, a química analítica tem ocupado um lugar de destaque, já que, dentro do contexto das ciências exatas, existe uma grande necessidade de contar com ferramentas analíticas para determinação quantitativa e qualitativa de espécies químicas contidas em ampla variedade de amostras. Desta forma, a química analítica tem contribuído para o estabelecimento de metodologias analíticas cada vez mais sensíveis, seletivas e confiáveis. Esta procura por novas alternativas tem propiciado o desenvolvimento de muitas técnicas analíticas instrumentais, grande parte das quais, além de representar um sólido avanço da própria área, tem sido fundamental para o desenvolvimento de muitos campos da ciência.

Dentro do contexto das novas técnicas analíticas instrumentais, a espectrometria de Fluorescência de Raios-X (FRX) ocupa um lugar de destaque, principalmente para aquelas áreas em que a obtenção de rápido perfil de constituintes metálicos e não-metálicos é indispensável. Exemplos clássicos disto são as aplicações industriais ${ }^{1}$, que freqüentemente requerem rápidas rotinas analíticas para controle de qualidade de seus produtos, assim como as análises exploratórias utilizadas em geologia ${ }^{2,3}$, arqueologia ${ }^{4}$, $\operatorname{artes}^{4,5}$, ciência dos materiais ${ }^{6,7} \mathrm{e}$ até análises in vivo ${ }^{8,9}$. Grande parte destas determinações são extremamente facilitadas por FRX, graças a um conjunto favorável de características ${ }^{10}$ não usuais, dentre as quais destacam-se: a) capacidade para a realização de determinações multielementares simultâneas (tipicamente, de sódio até urânio), b) capacidade para análise qualitativa e quantitativa, c) operação com amostras sólidas e líquidas, d) apresentação de caráter não-destrutivo, e) insensibilidade à forma química em que as espécies de interesse se encontram.

\section{ESPECTROMETRIA DE FLUORESCÊNCIA DE RAIOS-X (FRX)}

\section{Fundamentos}

A FRX pode ser classificada como uma técnica de emissão atômica, fundamentada no efeito fotoelétrico. Quando um átomo é submetido a um processo de irradiação utilizando-se uma fonte de raios-X (tubo de raios-X, indução por partícula, radioisotópos naturais, luz síncrotron, etc ), um elétron pode ser ejetado das camadas eletrônicas mais internas (efeito fotoelétrico, vide Figura 1A). Para estabilização desta forma excitada, elétrons das camadas eletrônicas mais externos caem rapidamente para as vacâncias geradas, liberando a diferença de energia $(\Delta \mathrm{E})$ existente entre os dois níveis de energia envolvidos (Figura $1 \mathrm{~B}$ ). Como este processo envolve níveis de energia que são característicos de cada elemento, a radiação emitida para cada transição é também característica. Desta maneira, a energia da radiação emitida pode ser diretamente utilizada na identificação da espécie em questão. Por outro lado, como a intensidade da radiação emitida é diretamente proporcional à concentração da espécie, a técnica também fornece informações que podem ser utilizadas para fins quantitativos ${ }^{11,12}$.

Uma representação esquemática dos níveis de energia atômicos, das transições possíveis e das suas respectivas denominações, é apresentada na Figura 2.

\section{Aplicações}

Em função das características favoráveis mencionadas anteriormente, a utilização da FRX em rotinas de análise química deveria ter experimentado um crescimento muito mais significativo do que é possível verificar na literatura recente. A técnica oferece indiscutíveis vantagens em relação a outras técnicas espectroscópicas (ex.: Espectrometria de Absorção Atômica (AA) e Espectrometria de Emissão de Plasma Indutivamente Acoplado (ICP)), especialmente naqueles casos em que, em função das características físicas da amostra (ex.: sólido ou líquido muito viscoso), as técnicas concorrentes precisam de operações preliminares de abertura ou tratamento. Em situações nas quais pretende-se analisar uma amostra totalmente desconhecida, a técnica de FRX também mostra-se mais vantajosa, principalmente em função de permitir uma rápida avaliação qualitativa dos constituintes da matriz ${ }^{13}$.

Existem, no entanto, dois fatores de grande importância que se constituem como os principais limitantes para a definitiva consolidação da técnica de FRX convencional como ferramenta analítica de primeira grandeza. São elas, a baixa sensibilidade da técnica e as denominadas interferências de matriz, sendo esta última o objeto de estudo deste trabalho. 

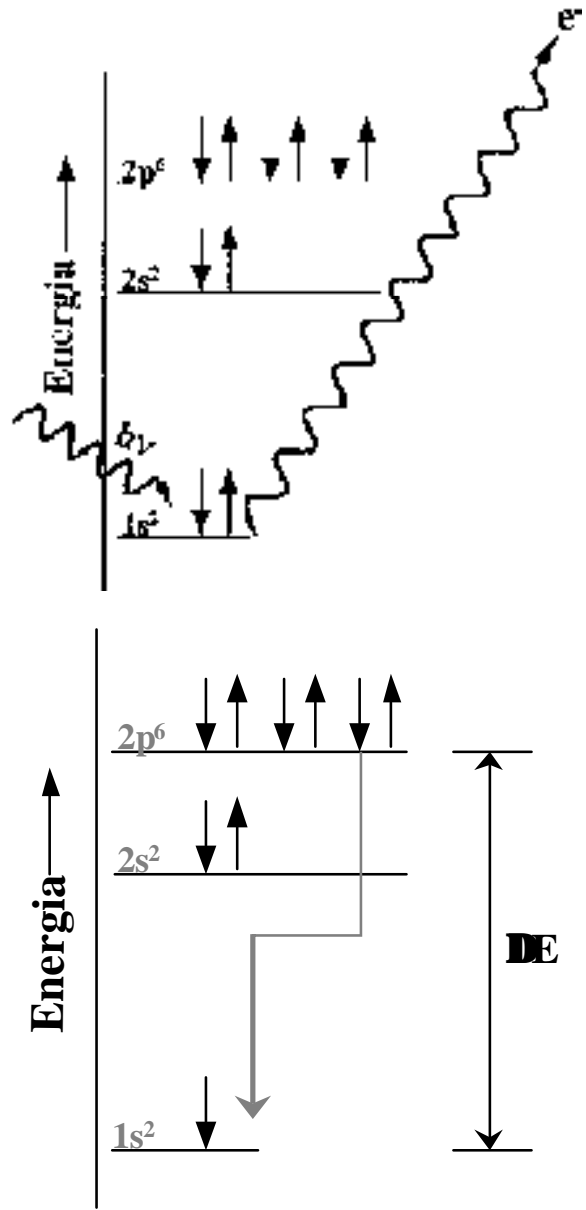

Figura 1. Representação dos Fundamentos da Espectrometria de Fluorescência de Raios-X. (A) Efeito Fotoelétrico ( $h \mathrm{~V}$ : energia contida na radiação eletromagnética originada na fonte de excitação); (B) Emissão de Raios-X provenientes de transições eletrônicas subsequentes.

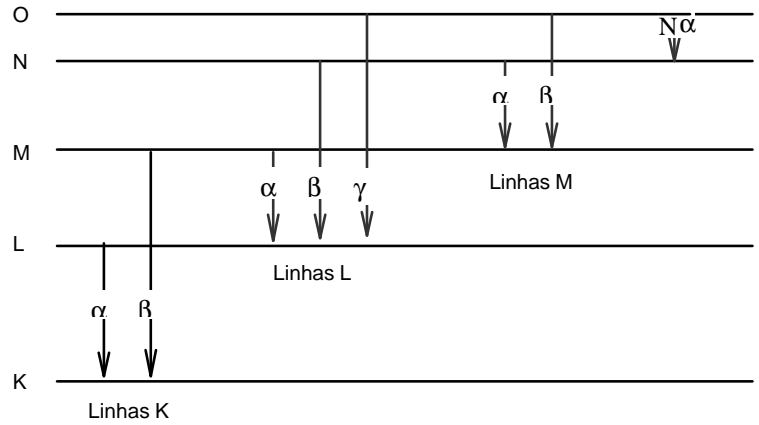

Figura 2. Diagrama das transições e suas respectivas denominações.

\section{Interferências}

Uma vez que a técnica envolve a participação de elétrons das camadas mais internas, tanto a energia como a intensidade da radiação emitida são insensíveis à forma química em que o elemento se encontra. Esta característica, apesar de impedir a especiação química de elementos, contribui para a ausência de interferências químicas, fator que diferencia bastante a FRX de outras técnicas espectroscópicas que envolvem transições de elétrons de valência. Nestes casos, a forma química em que o elemento de interesse se apresenta é que define a natureza da interação com a radiação.

Em geral, a técnica de FRX é susceptível a três tipos de interferências: a. Física da Matriz ${ }^{14}$ : Como em toda técnica analítica, grande parte dos problemas de interferência deste tipo é resultado de amostras pouco homogêneas. No caso da FRX, este fenômeno é agravado pelo efeito do tamanho das partículas presentes em amostras e padrões. Esta diferença de granulometria dificulta a irradiação homogênea, gerando um sombreamento nas partículas menores, quando estas encontram-se nas proximidades de partículas significativamente maiores. No entanto, este tipo de interferência pode ser minimizada efetuando-se processos de trituração, peneiramento e posterior homogeneização da amostra.

b. Espectral. Representada pela sobreposição de linhas de emissão no espectro de FRX. Os problemas mais graves envolvem a interferência da principal linha de emissão do elemento de interesse e outra linha de emissão principal (Figura $3 \mathrm{~A}$, As-K $\alpha: 10,530 \mathrm{keV}$ e $\mathrm{Pb}-\mathrm{L} \alpha: 10,550 \mathrm{eV}$ ) ou secundária (Figura $3 \mathrm{~B}$, linha $\mathrm{Ka}$ de elemento com número atômico $\mathrm{Z}$, sofrendo sobreposição por linha $\mathrm{K} \beta$ de elemento de número atômico Z-1), de outro elemento presente na matriz. Em função da relativa simplicidade do espectro de emissão de raios- $x$ e das características do sistema instrumental, os problemas derivados deste tipo de interferência costumam ser pouco numerosos ${ }^{10}$. Em geral, a técnica permite a determinação simultânea de misturas bastante complexas (ex.: zircônio-háfnio, lantanídios, etc), difíceis de serem determinadas através de outras técnicas espectroscópicas em função do elevado grau de semelhança nas propriedades químicas destas espécies. Quando este tipo de interferência se manifesta, várias alternativas podem ser utilizadas para contornar os seus efeitos. Dentre as mais importantes destacam-se: a) escolha de uma linha espectral alternativa, b) processos de separação química, c) métodos matemáticos (deconvolução espectral ${ }^{15}$, calibração multivariada ${ }^{16}$, etc).
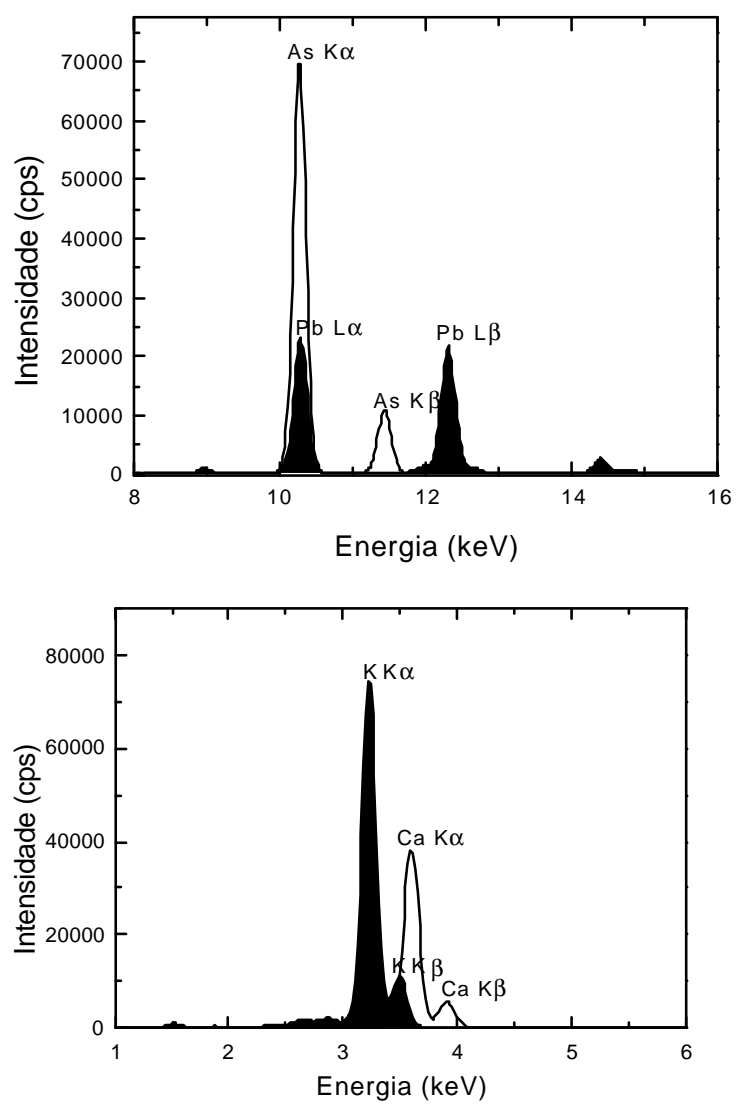

Figura 3. Representação das Interferências Espectrais presentes na análise por FRX 
c. Efeito de Matriz ${ }^{14}$ : Conhecido também como Efeito Interelementos, este tipo de interferência é causada basicamente por fenômenos de absorção ou intensificação do sinal de emissão, por parte dos outros elementos que compõem a matriz amostral.

\section{ABSORÇÃo}

O efeito de absorção pode se manifestar de duas maneiras, denominadas primária e secundária. Em ambos os casos, a quantidade de radiação absorvida por cada espécie interferente é uma função da capacidade particular de cada elemento, representada pelo coeficiente de absorção de massa.

A absorção primária é decorrente da absorção de fótons provenientes da fonte primária, por todos os elementos que compõem a matriz amostral. Assim, a distribuição intensidade vs comprimento de onda dos fótons disponíveis para a excitação do elemento de interesse pode ser modificada pela competição com outros elementos presentes na matriz.

Uma representação esquemática deste processo de interferência é apresentado na forma de diagramas de intensidade $v s$ comprimento de onda ${ }^{14}$. Na Figura 4A, mostra-se a distribuição de fótons primários disponíveis para a excitação dos elementos $\mathrm{A}$ e B (área destacada), as curvas de absorção destas espécies (linhas contínuas) e as suas respectivas linhas de emissão (linhas onduladas). Na Figura seguinte (4B), representa-se a porção da radiação primária disponível para excitação da espécie A. Obviamente que, em função da significativa absorção por parte da espécie $\mathrm{B}$, a radiação disponível é menor que o contínuo fornecido originalmente pela fonte primária. A situação análoga para o elemento B é apresentada na Figura 4C.

$\mathrm{O}$ processo de absorção primária reduz a intensidade do subseqüente processo de emissão, provocando uma significativa diminuição na intensidade da radiação fornecida pela fonte primária para excitação do elemento de interesse.

$\mathrm{O}$ efeito de absorção secundária está relacionado com a absorção da radiação característica emitida pelo elemento de interesse, por parte de outros elementos presentes na matriz.

$\mathrm{O}$ fenômeno de absorção secundária fica claramente ilustrado no esquema apresentado na Figura 4A. Se for considerado que, nesta mistura, o elemento A representa a espécie de interesse e o elemento B o interferente, é possível verificar que grande parte da emissão de A será absorvida pela espécie B. Nesta situação, a interferência poderá inviabilizar a determinação. Na situação inversa a interferência não é muito significativa, uma vez que o coeficiente de absorção de massa de A é praticamente desprezível no comprimento de onda da emissão de B.

\section{Intensificação da Fluorescência}

Ao contrário do fenômeno de Absorção, o da Intensificação da Fluorescência contribui com o aumento de sinal de emissão do elemento de interesse. Este fenômeno é produzido quando elementos presentes na matriz podem emitir radiação de energia igual ou superior à necessária para a excitação do elemento de interesse. No exemplo apresentado na Figura 4, fica evidente que a emissão do elemento A, por corresponder a uma energia muito próxima ao máximo de absorção do elemento $\mathrm{B}$, poderá contribuir significativamente com a excitação do mesmo. Sendo assim, a possibilidade de acontecer um incremento no sinal de emissão de $\mathrm{B}$, em relação a sua emissão na ausência de A, é muito provável.

Interferências deste tipo podem envolver mais do que um elemento interferente. Um esquema representativo do processo, envolvendo a ausência deste fenômeno, como também a participação de um e dois interferentes, é apresentado na Figura 5.

\section{MÉTODOS DE CORREÇÃO}

Em decorrência dos efeitos de matriz citados anteriormente, é muito comum que a medida de intensidade de emissão de raios- $\mathrm{X}$
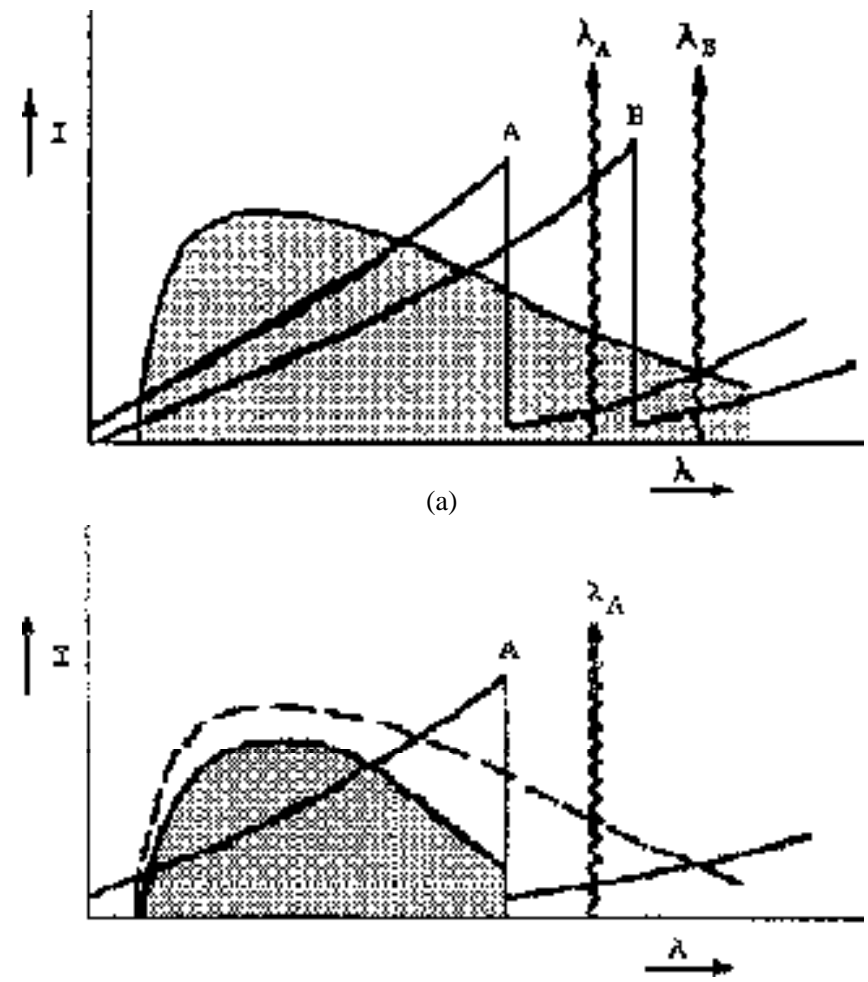

(b)

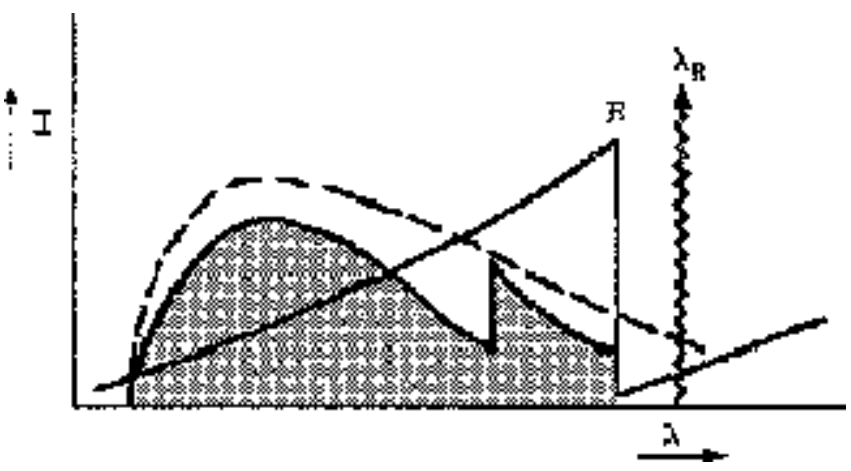

(c)

Figura 4: Espectro Primário Total disponível para Excitação. X-Ray Spectrometry; Ron Jenkins; Canadá, 1988. "Translate by permission of John Wiley \& Sons, Inc. All rights reserved"

característica de um elemento de interesse não se correlacione linearmente com a sua concentração. Como os efeitos interelementares são sistemáticos e previsíveis na análise por $\mathrm{FRX}^{17}$, inúmeras metodologias para correção foram propostas desde a década de 50. Dentre as mais importantes podem-se citar: a) sistema de adição e diluição de padrão ${ }^{18}$, b) métodos baseados no espalhamento da radiação ${ }^{19,20}$, c) sistema de calibração ${ }^{21}$ com padrões certificados de composição similar às amostras, d) métodos matemáticos (coeficientes de influência, parâmetros fundamentais, calibração multivariada e redes neurais).

Para avaliação de cada um dos métodos matemáticos citados, utilizar-se-ão como exemplos trabalhos publicados com a análise de um sistema clássico que apresenta fortes efeitos interelementos, ou seja, a análise quantitativa de ligas de $\mathrm{Fe}-\mathrm{Ni}-\mathrm{Cr}$.

\section{PRIMEIROS MÉTODOS MATEMÁTICOS}

Em 1955, Sherman ${ }^{22}$ propôs equações matemáticas baseadas em parâmetros fundamentais e instrumentais que permitiam o cálculo das intensidades de raios-X emitidas pelos elementos 


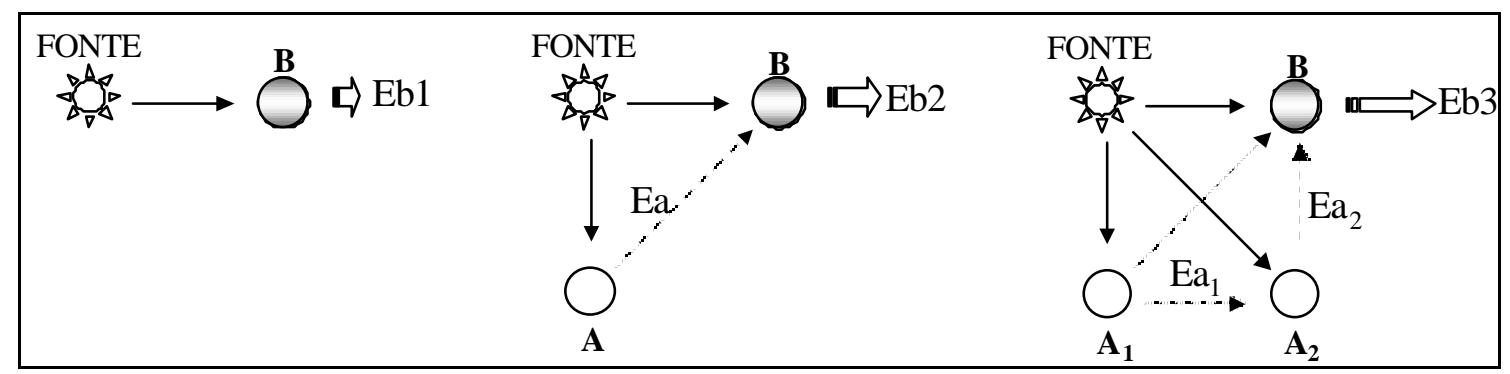

Figura 5. Representação do fenômeno de intensificação de fluorescência. A: elementos interferentes; B: elemento de interesse; Ea: emissão de $\boldsymbol{A}$ com energia suficiente para excitar $\boldsymbol{B}$ e intensificar o sinal do analito; $\boldsymbol{E} \boldsymbol{b}$ : emissão do analito $\left(E b_{1}<E b_{2}\right.$ e $\left.E b_{3}\right)$

que compunham uma determinada amostra de composição conhecida, quando irradiada com um feixe de raios-X policromático. Infelizmente, em decorrência da elevada complexidade das equações teóricas propostas e da não disponibilidade de ferramentas computacionais, o método tornou-se impraticável para os analistas da época ${ }^{23}$. Em função disto, procurou-se condensar o efeito interelemento total em uma simples constante, denominada como coeficiente de influência (CI). Assim, entre os anos de 1950 e 1980 surgiu uma série de novos algoritmos matemáticos, os quais podem ser classificados como ${ }^{23,24}$ :

a) Fundamental: coeficiente definido e derivado explicitamente em termos de parâmetros fundamentais (definidos mais adiante) e instrumentais.

b) Semi-Empírico: coeficiente definido teoricamente e derivado de aproximações das equações fundamentais.

c) Empírico: coeficiente definido experimentalmente (análise de regressão) e derivado de aproximações das equações fundamentais.

O grande avanço nos métodos matemáticos aconteceu em 1968, ano em que Criss e Birks ${ }^{25}$ propuseram a técnica de Parâmetros Fundamentais (PF), metodologia que foi considerada como o estado da arte para correção dos efeitos de matriz. Dentre outros fatores, a utilização de cálculos matemáticos iterativos e a não necessidade de utilização de padrões, foram pontos fortes que incentivaram e ampliaram a sua aplicação. Sua principal deficiência, no entanto, está localizada nas incertezas presentes nos coeficientes de absorção de massa e rendimentos fluorescentes do analito, dados necessários para o desenvolvimento da equação fundamental ${ }^{23,26}$.

\section{Coeficiente de Influência Empírico}

Este método está baseado na determinação de coeficientes numéricos capazes de corrigir o efeito de cada elemento da matriz, na resposta do elemento de interesse. Assim, se o efeito de matriz do elemento interferente $j$ em outro elemento da amostra $i$ puder ser expresso como uma constante, $\mathrm{a}_{i j}$, é possível escrever a seguinte correlação de Intensidade (I) como função de Concentrações $(C)^{27}$ :

$\mathrm{Ii}=\frac{\mathrm{Ci}}{\sum \mathrm{a}_{\mathrm{ij}} \mathrm{Cj}}$

A validade desta equação depende basicamente de 3 considerações (aproximações): a) a amostra é homogênea, com espessura infinita (espessura da amostra a partir da qual não há transmissão) e superfície plana, b) a radiação primária é monocromática (se o feixe for policromático, poderá ser considerado como equivalente a uma radiação primária monocromática de alguns comprimentos de onda específicos), c) o efeito de intensificação de fluorescência secundária dentro da amostra é considerada como absorção negativa ${ }^{17,27}$.

As duas últimas considerações são fisicamente incorretas, mas são aceitáveis se o conjunto de coeficientes de influência é determinado utilizando-se padrões com uma faixa pequena de concentração e composição bastante próxima da amostra de interesse ${ }^{27}$.

Estes coeficientes podem ser determinados a partir da Eq. 01, resolvendo-se um conjunto de equações simultâneas. Neste ponto, surge uma limitante matemática, pois o número de incógnitas $\left(\mathrm{a}_{i j}\right)$ dever ser igual ou inferior ao número de equações do sistema. Para evitar qualquer tipo de problema e garantir que o sistema tenha solução, é necessário ter um número de padrões igual ou superior ao número de elementos que se deseja quantificar ${ }^{27}$.

Para um sistema contendo 2 analitos (A e B) podem ser determinados 4 coeficientes de influência $\left(\mathrm{a}_{A A}, \mathrm{a}_{A B}, \mathrm{a}_{B B}, \mathrm{a}_{B A}\right)$, utilizando-se os dados de concentração e sinal analítico de 2 padrões (1 e 2).

$$
\begin{aligned}
& \mathrm{C}_{\mathrm{A} 1} / \mathrm{I}_{\mathrm{A} 1}=\mathrm{A}_{A A} \mathrm{C}_{\mathrm{A} 1}+\mathrm{A}_{A B} \mathrm{C}_{\mathrm{B} 1} \\
& \mathrm{C}_{\mathrm{A} 2} / \mathrm{I}_{\mathrm{A} 2}=\mathrm{A}_{A A} \mathrm{C}_{\mathrm{A} 2}+\mathrm{A}_{A B} \mathrm{C}_{\mathrm{B} 2} \\
& \mathrm{C}_{\mathrm{B} 1} / \mathrm{I}_{\mathrm{B} 1}=\mathrm{A}_{B B} \mathrm{C}_{\mathrm{B} 1}+\mathrm{A}_{B A} \mathrm{C}_{\mathrm{B} 1} \\
& \mathrm{C}_{\mathrm{B} 2} / \mathrm{I}_{\mathrm{B} 2}=\mathrm{A}_{B B} \mathrm{C}_{\mathrm{B} 2}+\mathrm{A}_{B A} \mathrm{C}_{\mathrm{B} 2}
\end{aligned}
$$

A análise da amostra desconhecida pode então ser obtida a partir da mesma Eq.01 escrita de uma outra forma, utilizando a resposta instrumental e os 4 coeficientes recém determinados.

$$
\begin{aligned}
& \left(\mathrm{I}_{\mathrm{A}} \mathrm{a}_{A A}-1\right) \mathrm{C}_{\mathrm{A}}+\mathrm{I}_{\mathrm{A}} \mathrm{a}_{A B} \mathrm{C}_{\mathrm{B}}=0 \\
& \mathrm{I}_{\mathrm{B}} \mathrm{a}_{B A} \mathrm{C}_{\mathrm{A}}+\left(\mathrm{I}_{\mathrm{B}} \mathrm{a}_{B B}-1\right) \mathrm{C}_{\mathrm{B}}=0 \\
& \mathrm{C}_{\mathrm{A}}+\mathrm{C}_{\mathrm{B}}=1
\end{aligned}
$$

Um novo sistema de equações é obtido e, como as Eqs. 04a e 04b não são mutualmente independentes, é necessário deduzir uma outra relação válida para o problema. Sabendo que a amostra de interesse é constituída apenas por dois elementos, a Eq. 04c pode ser considerada.

É importante destacar que as constantes calculadas intrinsecamente carregam informações de parâmetros instrumentais em que foram obtidos os espectros (fonte de raios-X ou alvo, voltagem e tipo de espectrômetro). Obviamente, qualquer modificação instrumental introduzida poderá implicar na necessidade de uma nova determinação destes coeficientes empíricos.

Apesar das dificuldades e aproximações impostas pelo método de correção por coeficientes empíricos, seu fundamento básico proporcionou e continua a proporcionar a criação de um elevado número de novos algoritmos. Isto tem contribuído para gerar uma grande confusão quanto ao conceito e à nomenclatura dos coeficientes de influência (sejam eles empíricos ou teóricos).

Criss e Birks $^{25}$ demonstraram a viabilidade da determinação quantitativa de ligas de $\mathrm{Fe}-\mathrm{Ni}-\mathrm{Cr}$ através da metodologia de coeficientes de influência empírico. Como estes coeficientes foram determinados pela solução de um conjunto de equações 
simultâneas, o número de amostras para a etapa de calibração foi igual ao número de analitos de interesse. Para efeito de comparação, foram estudados dois procedimentos de obtenção dos CI usando a liga de $\mathrm{Fe}, \mathrm{Ni}$ e $\mathrm{Cr}$ como exemplo. No primeiro procedimento, utilizaram-se padrões que cobriam uma ampla faixa de concentração $(\mathrm{Cr}$ : $0-21,6 \%$, Fe: 6-86,1\%, Ni: $0,16-78,1 \%)$; no segundo, utilizaram-se apenas padrões com teores próximos àqueles encontrados nas amostras problema (Cr: 17,2-17,9\%, Fe: 64,8-72,67\%, Ni: 7,23-12,8\%). Os resultados deste estudo demonstraram claramente que a utilização de padrões de composição mais próxima não leva necessariamente à obtenção de CI mais representativos do fenômeno de interferência. Desta forma, ambas as metodologias levaram à obtenção de erros médios relativos comparáveis (Cr: 2,4\%, Fe: $3 \%$ e Ni: $3,6 \%)$ e aceitáveis para a maioria dos objetivos da análise.

Grande parte do sucesso da utilização de CI na resolução de problemas de interferência interelementos está relacionada com a obtenção de coeficientes numéricos confiáveis e representativos do processo interferente, o que é extremamente dificultado em análises que envolvem matrizes complexas ou ainda de composição não claramente definida.

\section{Parâmetros Fundamentais}

Esta metodologia permite calcular a composição analítica de uma amostra, a partir da medida de intensidade da linha de emissão do analito e valores tabelados de três parâmetros fundamentais: distribuição espectral primária (fonte), coeficiente de absorção (fotoelétrico e de massa), e rendimento de fluorescência ${ }^{17}$. O coeficiente de absorção $(\mu)$ é uma constante que relaciona a perda de intensidade de fluorescência quando a radiação atravessa uma amostra, dividido pela espessura da mesma. No entanto, o coeficiente de absorção de massa $\left(\mu_{\mathrm{m}}\right)$ que é uma função de $\mu$ dividido pela densidade do material (elemento), é uma constante mais útil ${ }^{12,27}$.

A equação abaixo mostra a expressão matemática necessária para aplicação do processo de PF, considerando-se a excitação da amostra através de radiação monocromática. Utilizando-se fonte policromática na análise de amostras multicomponentes, a expressão torna-se bastante complexa, o que leva à utilização de recursos computacionais ${ }^{10}$.

$$
\mathrm{I}_{\mathrm{L}}=\mathrm{I}_{0} \omega_{\mathrm{A}} \mathrm{g}_{\mathrm{L}} \frac{\mathrm{r}_{\mathrm{A}}-1}{\mathrm{r}_{\mathrm{A}}} \frac{\mathrm{d} \Omega}{4 \pi} \frac{\mathrm{C}_{\mathrm{A}} \mu_{\mathrm{A}}\left(\lambda_{\text {prim }}\right) \cos \sec \varphi}{\mu_{\mathrm{M}}\left(\lambda_{\text {prim }}\right) \cos \sec \varphi+\mu_{\mathrm{M}}\left(\lambda_{\mathrm{L}}\right) \cos \sec \psi}
$$

$\mathrm{I}_{\mathrm{L}}$...Intensidade da linha do analito $\mathrm{A} ; \mathrm{I}_{\mathrm{O}}$...Intensidade do feixe primário em efetivo comprimento de onda $\lambda_{\text {prim }} ; \lambda_{\text {prim....Efetivo }}$ comprimento de onda do raio-X primário; $\lambda_{\mathrm{L}}$...Comprimento de onda da linha do analito $\mathrm{A} ; \omega_{\mathrm{A}} \ldots$ Rendimento de fluorescência do elemento $\mathrm{A} ; \mathrm{g}_{\mathrm{L}}$...Valor fracional da linha $\mathrm{L}$ do analito em sua série; $\mathrm{r}_{\mathrm{A}}$...Razão do borda de absorção do elemento $A ; d \Omega / 4 \pi$...Valor fracional do raio-X fluorescente dirigido ao detector; $\mathrm{C}_{\mathrm{A}}$...Concentração do elemento $\mathrm{A}$; $\mu_{\mathrm{A}}\left(\lambda_{\text {prim }}\right)$...Coeficiente de absorção de massa de A para $\lambda_{\text {prim }}$; $\mu_{\mathrm{M}}\left(\lambda_{\text {prim }}\right)$...Coeficiente de absorção de massa da matriz para $\lambda_{\text {prim; }} \mu_{M}\left(\lambda_{L}\right)$...Coeficiente de absorção de massa da matriz para $\lambda_{\mathrm{L}} ; \varphi$...Ângulo de incidência do feixe primário; $\psi$..Ângulo de saída do feixe fluorescente

Em contraste com o método de correção por coeficientes empíricos, o método por parâmetros fundamentais apenas assume que a amostra é homogênea, apresenta espessura infinita e tem uma superfície razoavelmente plana.

Apesar da possibilidade de analisar amostras sem a utilização de padrões, o método ganha maior confiabilidade se os diversos parâmetros que fazem parte da expressão matemática são obtidos a partir de padrões adequados. O cálculo envolve duas etapas fundamentais: a calibração e a previsão. $\mathrm{Na}$ primeira etapa, a equação de PF é utilizada para prever a intensidade das linhas características do padrão de calibração. Os cálculos são realizados especificamente para o espectrômetro em que as medidas estão sendo realizadas, uma vez que a equação de PF considera aspectos de geometria, fonte e condições instrumentais. As intensidades teoricamente calculadas são relacionadas com as intensidades medidas, sendo esta última corrigida para cada linha característica. Esta correção é feita graficando-se intensidades líquidas (experimentais) vs intensidades calculadas, sendo que a inclinação da curva corresponde ao fator proporcional que deve ser utilizado na correção ${ }^{26}$.

$\mathrm{Na}$ etapa da previsão é necessário estimar primeiramente uma composição aproximada da amostra. Isto geralmente é conseguido considerando-se a intensidade relativa das linhas de emissão detectadas para cada elemento presente na matriz e assumindo que o total emitido corresponde a uma composição de $100 \%$ (ou algum outro total se os constituintes menores forem ignorados) $)^{27}$.

A partir desta equação, um programa calcula quais intensidades deveriam ser observadas para a suposta composição, compara-as com os valores medidos, ajusta a composição assumida e calcula o novo conjunto de intensidades esperadas. Este processo de iteração é repetido automaticamente até que a composição suposta dê uma intensidade de raios-X que corresponde ao valor medido dentro de algumas faixas de erro. A composição que satisfaz esta relação é mostrado como o resultado da análise ${ }^{17}$.

Criss e Birks ${ }^{25}$ aplicam a técnica de PF para as amostras de ligas $\mathrm{Fe}-\mathrm{Ni}$-Cr com o intuito de comparar os resultados de ambas as metodologias aplicadas (CI e PF), na correção dos problemas de interferência de matriz. Os autores optaram pela não utilização de padrões (que seria teoricamente a pior situação de análise) e mesmo assim, obtiveram para Fe $(1,2 \%)$ e $\mathrm{Ni}(1,5 \%)$ uma exatidão melhor que o método de CI. A exceção ocorreu para a quantificação do $\mathrm{Cr}(7,0 \%)$, cujo resultado foi explicado justamente pelas incertezas presentes nos coeficientes de absorção de massa e rendimentos fluorescentes do analito.

Christensen e $\operatorname{Pind}^{28}$ propuseram algumas aproximações no método de parâmetros fundamentais para a análise de ligas de $\mathrm{Fe}-\mathrm{Ni}$-Cr usando equipamento de fluorescência de raios-X de energia dispersiva, com excitação por alvo secundário. A primeira aproximação realizada considerou que a fonte instrumental era monocromática e, posteriormente, para incluir no modelo as contribuições da intensificação de fluorescência terciária, os autores utilizaram uma aproximação sugerida em outro traba1 lho $^{29}$ (ambas as considerações facilitam os cálculos). Foram analisadas 6 amostras certificadas de ligas e, de acordo com os resultados apresentados, é possível verificar que, para a quantificação de $\mathrm{Fe}$, Ni e $\mathrm{Cr}$, a influência do processo de intensificação de fluorescência terciária é pouco representativa. A exatidão do método fica em $5 \%$ para a maior parte dos elementos (tanto para os componentes majoritários como minoritários).

A literatura recente ${ }^{7}$ mostra uma diversificada aplicação da metodologia de PF na análise de matrizes complexas via FRX. Este processo, além de constituir uma boa alternativa para contornar os problemas devidos a absorção, permite, na maioria dos casos, minimizar significativamente as interferências devidas a intensificação. Talvez, um dos poucos pontos fracos desta proposta esteja representado pela sua inabilidade na correção de interferências espectrais. Este tipo de interferência, embora não muito comum para amostras simples determinadas via FRX, costuma se manifestar seriamente em matrizes mais complexas.

\section{MÉTODOS QUIMIOMÉTRICOS}

Métodos de calibração multivariada ${ }^{30}$ têm sido cada vez mais utilizados em química analítica, principalmente quando os componentes presentes numa mistura necessitam ser determinados, mas a informação analítica disponível não apresenta seletividade. Isto é, quando em uma mistura não é possível 
identificar os componentes individuais, a partir da resposta instrumental. A base da calibração multivariada é estabelecer uma relação entre duas matrizes (ou blocos) de dados químicos, quando houver uma dependência entre as propriedades que descrevem cada uma delas.

A calibração multivariada consiste basicamente de duas fases: a calibração e a previsão. Na fase de calibração, "n" espectros para um conjunto de amostras com composição conhecida são obtidos em "p" valores de energia diferentes, formando uma matriz X, com "n" linhas e "p" colunas. Também uma matriz Y com os valores de concentração pode ser formada contendo " $\mathrm{n}$ " linhas, correspondendo às diferentes amostras, e "q" colunas, indicando o número de diferentes metais presentes nas amostras.

O próximo passo é desenvolver um modelo matemático apropriado (determinando o vetor dos coeficientes de regressão - b) que melhor possa reproduzir Ycal a partir dos dados da matriz Xcal (Eq.06). Esse modelo é utilizado na fase de previsão (com um conjunto teste) para estimar as concentrações (Yprev) dos constituintes de novas amostras, a partir de seus espectros (Xteste) (Eq.07). Como estas metodologias trabalham com matrizes de dados, o processo de isolar o fator $\mathbf{Y}$ da Eq.06 para obtenção da Eq.07, implica na utilização da matriz transposta de $\mathbf{X}$, ou seja, (Xteste) ${ }^{\mathbf{t}}$.

Xcal $=\mathbf{b} *$ Ycal

Yteste $=(\text { Xteste })^{\mathrm{t}} * \mathbf{b}$

Os dados para a calibração multivariada podem ser organizados conforme é mostrado na Figura 6. Os valores de emissão (cps: contagens por segundo) dos espectros, a cada valor de energia, são as variáveis independentes, e as concentrações dos metais nas amostras, as variáveis dependentes.

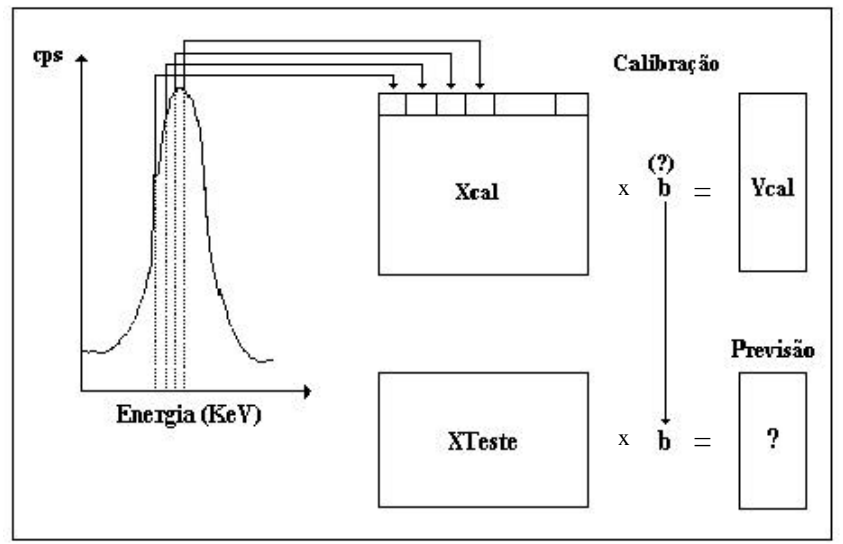

Figura 6. Organização dos dados para Calibração Multivariada.

\section{Método PLSR}

Existem várias metodologias para determinar este vetor de coeficientes de regressão (b), e uma das mais difundidas é a Regressão de Mínimos Quadrados Parciais (PLSR) que está baseada na Análise de Componentes Principais (PCA) ${ }^{31}$.

$\mathrm{O}$ método PCA decompõe a matriz de dados $\mathbf{X}$, construindo-se um novo sistema de eixos (denominados componentes principais). Isto possibilita que as amostras possam ser representadas e visualizadas em um número menor de dimensões, sem perda de informação analítica relevante. Com a obtenção de um novo sistema de eixos, a parte não modelada de $\mathbf{X}$ é considerada como uma matriz de erros $(\mathbf{E})$, as novas coordenadas da amostra são denominadas "scores" (t ou u), e o peso que cada variável antiga contribui para formar as componentes principais é chamado "loadings" (p ou q) $)^{31}$.

Simplificadamente, o método PLSR pode ser explicitado da seguinte forma. Tanto a matriz das variáveis independentes Xcal (Eq.08), como a das variáveis dependentes Ycal (Eq.09), sofrem esta decomposição matricial, sendo representadas na forma de "scores" e "loadings".

$$
\begin{aligned}
& \text { Xcal }=\mathbf{T P}^{\prime}+\mathbf{E} \\
& \text { Ycal }=\mathbf{U Q}^{\prime}+\mathbf{E}
\end{aligned}
$$

Posteriormente, realiza-se uma relação entre as duas matrizes de "scores" (Eq.10) de cada um dos blocos (variáveis independentes e dependentes), utilizando um modelo linear:

$\mathbf{U}=\mathbf{b} * \mathbf{T}$

A determinação correta do vetor $\mathbf{b}$, depende da otimização de dois fatores: a) escolha do número de componentes principais suficientes para explicar toda a informação analítica necessária para o problema e b) detecção de amostras anômalas com sua subsequente retirada (aquelas que apresentam um comportamento espectral diferenciado da média, e podem distorcer o modelo construído).

Obtido o vetor de regressão, é possível utilizá-lo para realizar as previsões de concentrações de novas amostras através de seus respectivos dados espectrais e empregando-se a Eq.07.

Os métodos de calibração multivariada têm sido utilizados com bastante freqüência e sucesso na resolução de problemas de interferência espectral, principalmente associados a outras técnicas analíticas.

Wang et $a l .{ }^{32}$ realizaram um trabalho exploratório envolvendo seleção de variáveis, e aplicação de PLSR e CI para análise de ligas de níquel constituída de $\mathrm{Fe}, \mathrm{Cr}, \mathrm{Ni}, \mathrm{Mn}$, Mo, Ti e $\mathrm{Si}$ por FRX. A seleção de variáveis foi realizada descartando-se os vetores coluna com pequena representatividade, obtidos através de Decomposição de Valor Singular ou eliminando-se regiões espectrais com limites pré-definidos. O modelo PLSR foi elaborado a partir de 15 padrões, utilizando-se autoescalamento como pré-processamento e cross validation leave-one-out como sistema de validação. Neste último procedimento, a calibração é repetida $n$ vezes ( $n=$ número de amostras), sendo que em cada oportunidade uma das amostras do conjunto de calibração é utilizada como amostra de previsão. Os resultados apresentados (teor de $\mathrm{Fe}, \mathrm{Cr}$, $\mathrm{Ni}$ e Mn) indicam que todos os modelos PLSR elaborados levam à obtenção de menores erros de previsão, em relação aos valores obtidos pelo método convencional de CI. Além disso, os resultados indicam que as etapas prévias ao desenvolvimento do modelo (ex. seleção de variáveis) é de fundamental importância para a obtenção de menores erros de previsão.

Os menores erros relativos obtidos por aplicação do modelo PLSR foram: Fe: 0,48\%, Cr: 1,04\%, Ni: $23 \%$ e Mn: 24,3\%. É conveniente salientar que, quando o método CI foi aplicado na análise das mesmas amostras, erros de previsão significativamente maiores foram encontrados (Fe: 69\%, Cr: 195\%, Ni: $12500 \%$, Mn: 2300\%).

\section{Redes Neurais}

As Redes Neurais Artificiais são definidas como um conjunto de métodos matemáticos e algoritmos computacionais projetados para imitar o processamento de informação e aquisição de conhecimento do cérebro humano ${ }^{33}$.

Elas são usualmente construídas por diversas camadas de neurônios, como mostrado na Figura 7. A primeira camada consiste de neurônios que simplesmente distribuem os valores de entrada no modelo, enquanto que a última camada produz a saída dos dados ${ }^{33}$.

Cada neurônio na NN é um mecanismo computacional simples, cuja entrada é associada a um peso, representado por um 


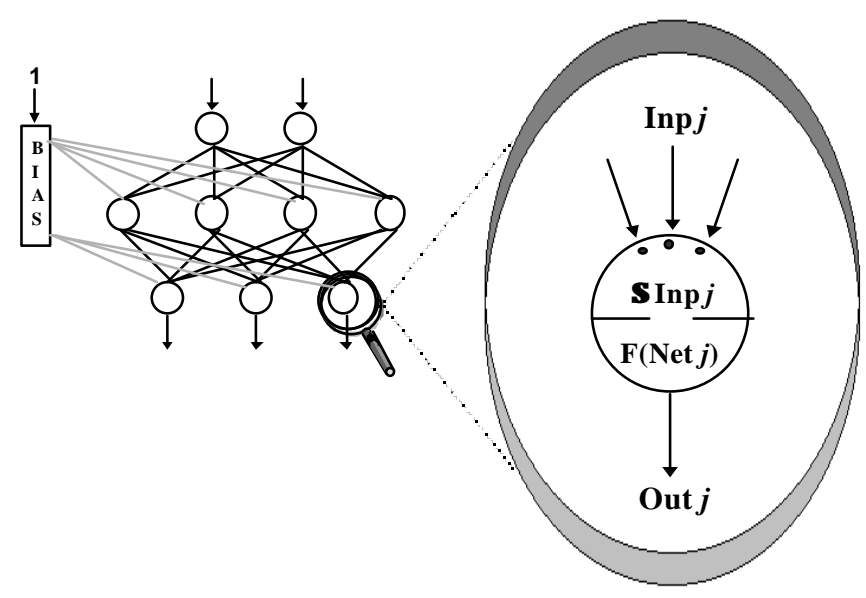

Figura 7. Representação da Rede Neural Artificial e da Unidade Estrutural (neurônio); Inp $\mathbf{j}$ : valor de entrada multiplicado por um peso; $\boldsymbol{\Sigma} n \boldsymbol{n}$ j: Somatória dos valores de Inp j; F(Net $\mathbf{j})$ : Função de Transferência (linear ou não-linear); Out j: valor de saída.

número de influência estimulante (positivo) ou inibidora (negativo) do sinal de entrada (em destaque). Os neurônios na camada escondida realizam a somatória de pesos dos valores de entradas $\left(\mathbf{N e t}_{\mathbf{j}}=\mathbf{\Sigma} \mathbf{I n} \mathbf{p}_{\mathbf{i}}\right)$, aplicando posteriormente uma função de transferência não-linear $\left(\mathbf{F}\left(\mathbf{N e t}_{\mathbf{j}}\right)\right)$. Os neurônios da camada de saída recebem os valores convertidos, provenientes dos neurônios da camada escondida, realizam a somatória de pesos e, para fins de classificação, estes neurônios podem realizar uma transformação não-linear. Contudo, para o propósito de calibração, os neurônios da camada de saída usualmente utilizam funções lineares ${ }^{34,35}$.

Definindo-se a topologia da NN a ser utilizada (número de neurônios, interconexões, camadas escondidas), a rede pode ser treinada pelo ajuste de seus pesos e bias (parâmetro adicional que ajusta os valores de Net $_{\mathrm{j}}$, confinando-os em um intervalo propício para aplicação da função de transferência). Inicialmente, todos os pesos são valores aleatórios, e o valor de entrada do bias é sempre 1. Durante o treinamento, uma série de amostras com seus correspondentes valores esperados de saída são apresentados à NN que, de maneira iterativa, ajusta ambos os parâmetros. No processo de treinamento, há primeiro uma modificação dos parâmetros entre as camadas de saída e escondida, usando-se posteriormente esta informação para modificar os parâmetros entre a camada escondida e a camada de entrada ${ }^{35,36}$.

O processo de treinamento mais conhecido é denominado back propagation, que compara a saída atual da rede neural e a desejada, ajustando os pesos da rede com a finalidade de diminuir o erro da saída, sempre na direção descendente do gradiente de menor erro.

As NN não detectam amostras anômalas por si só. Se nenhum método prévio for utilizado (p. ex. Análise por Componentes Principais) estas amostras são incluídas na construção do modelo, podendo prejudicar a habilidade de previsão. Por outro lado, existem casos em que esta metodologia é capaz de prever a resposta desejada, mesmo havendo uma pequena diferença na composição química, ou seja, possibilitando a realização de extrapolações no modelo construído.

$\mathrm{Na}$ literatura, existe menção à aplicação de NN em um número muito reduzido de trabalhos envolvendo a análise via Fluorescência de Raios- $X^{16,37-41}$.

Bos et al. ${ }^{38}$ aplicaram redes neurais para a análise de ligas de Fe-Ni-Cr via FRX, comparando os resultados com os obtidos por metodologias como Regressão Linear, Aproximação Semi-Linear e CI. O treinamento da rede foi realizado com
100 padrões (faixa de concentração - Fe: $50,48 \%$ a $98,17 \%$, Ni: $0,02 \%$ a $21,80 \%$ e $\mathrm{Cr}: 0,04 \%$ a $29,20 \%$ ) e utilização de back propagation. A arquitetura otimizada da rede consistiu de 12 neurônios na camada de entrada, 4 na intermediária (aplicando uma função de transferência sigmoidal) e 3 neurônios na camada de saída (12:4:3). A validação foi realizada através de cross validation leave-one-out para ganhar o máximo de informação dos dados disponíveis. Os resultados indicam que o modelo com NN proporciona erros relativos menores para a determinação de $\mathrm{Fe}(0,7 \%)$ e Ni $(29 \%)$, em relação aos resultados obtidos por meio dos outros procedimentos de correção estudados. Para a quantificação de $\mathrm{Cr}$, no entanto, melhores resultados foram obtidos por aplicação da metodologia de CI.

\section{OUTROS TIPOS DE APLICAÇÃO}

Conforme comentado anteriormente, o conjunto de vantagens obtidas pela utilização da técnica de FRX tem favorecido o desenvolvimento de uma grande diversidade de aplicações, principalmente em áreas como química, geologia e controle de qualidade de produtos industriais. Dentro destas áreas, percebe-se uma certa resistência à utilização de métodos matemáticos mais complexos, dando-se preferência a alternativas de natureza mais concreta (ex. métodos de adição ou diluição de padrão, utilização de padrões internos) ou a algorítimos matemáticos mais simples (ex. processos de regressão linear, regressão linear múltipla, deconvolução espectral).

Outras importantes aplicações dos métodos matemáticos de correção que podem ser encontradas na literatura recente são apresentadas a seguir (Tabela 1).

\section{CONCLUSÕES}

Em função de algumas dificuldades analíticas encontradas na analise quantitativa por FRX, principalmente decorrente dos efeitos de interferência de matriz, inúmeras metodologias químicas e matemáticas foram incorporadas ao dia-a-dia dos analistas.

Os métodos matemáticos convencionais mostraram-se muito úteis desde a década de 1950, principalmente considerando que muitas destas metodologias não necessitam de padrões para correção dos efeitos de matriz (coeficientes de influência teórico, parâmetros fundamentais). No entanto, sua base de cálculo pode tornar-se muito complicada e imprecisa, principalmente em função da complexidade da matriz amostral que deseja se analisar.

Por outro lado, os métodos quimiométricos baseiam-se na construção de modelos a partir de padrões de calibração. $\mathrm{Ou}$ seja, evitam o cálculo de coeficientes teóricos, relacionados à matriz amostral e às características geométricas e instrumentais, permitindo ao sistema obter modelos com maior habilidade de previsão. Além disso, uma outra grande vantagem destas metodologias em relação às convencionais, é a possibilidade de quantificar espécies mesmo na presença de dois problemas existentes em algumas análises por FRX: a interferência espectral e os efeitos interelementos.

No caso do PLSR, os maiores problemas estão associados a otimização do modelo, correspondentes as etapas de escolha do número de componentes principais necessários para explicar toda a informação analítica importante do sistema, assim como na detecção da presença de amostras anômalas.

As Redes Neurais apresentam a vantagem de construir modelos não lineares, que são uma das características dos efeitos interelementos. No entanto, a modelagem não detecta amostras anômalas, sendo necessário utilizar um método prévio para tal. 
Tabela 1. Recentes publicações relacionadas com a utilização de métodos matemáticos para correção do efeito de matriz nas análises por FRX.

\begin{tabular}{|c|c|c|c|c|}
\hline Técnica & Matriz Amostral & Descrição & Resultados & Ref. \\
\hline CI & $\begin{array}{c}\text { Solos e } \\
\text { Sedimentos }\end{array}$ & $\begin{array}{l}\text { CI determinado a partir de padrões } \\
\text { certificados (analitos: } \mathrm{Na}, \mathrm{Mg}, \mathrm{Al}, \mathrm{Si} \text {, } \\
\text { P, K, Ca, Ti, Mn, Fe, S) }\end{array}$ & $\begin{array}{l}\mathrm{Si}, \text { Al e Fe: altos erros } \\
\quad(\text { erros } \geq 13 \%)\end{array}$ & 42 \\
\hline $\mathrm{CI}$ & Amostra Geológica & $\begin{array}{l}\text { CI determinado por padrões puros de } \\
\text { cada um dos } 14 \text { elementos de interesse. }\end{array}$ & $\begin{array}{l}\text { Erro na determinação } \\
\text { de } \mathrm{Fe}_{2} \mathrm{O}_{3} \text { cai } 10 x \text { com o } \\
\text { emprego da técnica CI. }\end{array}$ & 43 \\
\hline $\mathrm{CI}$ & Minerais Silicatados & $\begin{array}{l}\text { CI determinado pelas estimativas nos } \\
\text { pontos de composição máxima. }\end{array}$ & $\begin{array}{l}\text { Erro relativo } \leq 5 \% \\
\uparrow\left[\mathrm{SiO}_{2}\right] \downarrow \text { Erro }\end{array}$ & 44 \\
\hline CI e PF & $\begin{array}{l}\text { Ligas } \\
\text { Fe-Ni-Cr }\end{array}$ & $\begin{array}{l}\text { Quantificação de } \mathrm{Fe}, \mathrm{Ni} \text { e } \mathrm{Cr} \\
\text { CI: determinado empiricamente } \\
\text { PF: sem padrões de calibração }\end{array}$ & $\begin{array}{l}\text { Fe e Ni: maior exatidão } \\
\text { Fempregando-se a } \\
\text { técnica de PF }\end{array}$ & 25 \\
\hline $\mathrm{CI}$ e PF & $\begin{array}{l}\text { Matéria orgânica, } \\
\text { biológica e solução aq. }\end{array}$ & $\begin{array}{l}\text { Quantificação de elementos } \\
\text { traços, principalmente, Ti, Ba, } \\
\text { As, Pb, K e Ca. PF: sem } \\
\text { padrões de calibração. } \\
\text { CI determinado empiricamente. }\end{array}$ & $\begin{array}{l}\text { Sol. Aquosa: } \mathrm{CI} \approx \mathrm{PF} \\
\mathrm{P} / \text { matriz complexa, PF é } \\
\text { ligeiramente melhor que CI }\end{array}$ & 45 \\
\hline $\mathrm{PF}$ & $\begin{array}{l}\text { Ligas } \\
\mathrm{Fe}-\mathrm{Ni}-\mathrm{Cr}\end{array}$ & $\begin{array}{l}\text { Quantificação de } \mathrm{Fe}, \mathrm{Ni} \text { e } \mathrm{Cr} \text { com } \\
\text { equipamento de EDXRF com } \\
\text { excitação por alvo secundário }\end{array}$ & $\begin{array}{l}\text { Análise de } 6 \text { amostras } \\
\text { certificadas com } \\
\text { exatidão } \approx 5 \%\end{array}$ & 28 \\
\hline $\mathrm{PF}$ & Solos (18 elementos) & $\begin{array}{c}\text { Calibração: } 7 \text { padrões certificados. } \\
\text { Previsão: } 10 \text { amostras certificadas. } \\
\text { Comparação do EDXRF fixo e portátil. }\end{array}$ & $\begin{array}{l}\text { Erro relativo } \leq 10 \% \\
\text { (s/ quantificar: } \mathrm{Cd}, \mathrm{Se} \\
\text { e } \mathrm{Hg} ; \text { falta de padrões) }\end{array}$ & 46 \\
\hline $\mathrm{PF}$ & Leite em pó (traços) & $\begin{array}{l}\text { Calibração: padrões de sais inorgânicos } \\
\text { puros. Previsão: } 2 \text { amostras certificadas }\end{array}$ & $\begin{array}{l}\text { Teor de } \mathrm{Zn}, \mathrm{As}, \mathrm{Br} \text { e } \mathrm{Rb} \\
\text { com boa exatidão }\end{array}$ & 47 \\
\hline $\mathrm{PF}$ & $\begin{array}{l}\text { Mineral Laterítico } \\
\text { (12 terras raras) }\end{array}$ & $\begin{array}{l}\text { Interferência espectral: solucionados } \\
\text { por deconvolução espectral; } \\
\text { e interferências de matriz: } \\
\text { procedimentos de extração } \\
\text { e PF (sem padrões) }\end{array}$ & $\begin{array}{l}\text { Erro relativo: } 1,5-24 \% \\
\text { LD: } 340 \mathrm{ppm} \text { (pesados) } \\
\quad \text { 4000ppm (leves) }\end{array}$ & 48 \\
\hline $\mathrm{PF}$ & $\mathrm{Au}$ em jóias & $\begin{array}{l}\text { Calibração: peças de Au de } 14 \text { à } 16 \mathrm{~K} \\
\text { (quantificados de acordo c/ ISO11426) }\end{array}$ & $\begin{array}{l}\text { Detecção: } \mathrm{Au}, \mathrm{Ag} \mathrm{e} \mathrm{Cu} \\
\text { Erro relativo } \approx 1 \%\end{array}$ & 49 \\
\hline PLSR & $S$ em grafite & $\begin{array}{l}\text { Calibração: } 8 \text { padrões sintéticos. } \\
\text { Modelo: log dos espectros; } \\
\text { pré-processamento: centrado na média; } \\
\text { contendo } 4 \text { componentes principais }\end{array}$ & $\begin{array}{l}\text { Exatidão }>5 \% \text { com } \\
\text { concentração de S } \\
\text { entre } 2-60 \%\end{array}$ & 50 \\
\hline PLSR e CI & Ligas de $\mathrm{Ni}$ & $\begin{array}{c}\text { Determinação de: } \mathrm{Fe}, \mathrm{Cr}, \mathrm{Ni} \text { e } \mathrm{Mn} \\
\text { Calibração: } 15 \text { padrões } \\
\text { Modelo: otimizado com cross } \\
\text { validation e autoescalamento dos dados }\end{array}$ & $\begin{array}{l}\text { PLSR melhor que CI } \\
\text { principalmente quando } \\
\text { empregada a seleção } \\
\text { de variáveis }\end{array}$ & 32 \\
\hline $\begin{array}{l}\text { PLSR, LR } \\
\quad \text { e CI }\end{array}$ & Ligas de $\mathrm{Fe}$ e $\mathrm{Cu}$ & $\begin{array}{l}\text { Calibração: ligas certificadas, } \\
15 \text { de Fe e } 20 \text { de } \mathrm{Cu} \text {. Uso de } \\
\text { seleção de variáveis e o PLSR: } \\
4 \text { componentes principais. }\end{array}$ & $\begin{array}{l}\text { C/ seleção variáveis: PLSR } \\
\text { melhor que OLS } \\
\text { e comparável a CI. }\end{array}$ & 51 \\
\hline $\begin{array}{l}\text { PLSR, LR } \\
\text { e MLR }\end{array}$ & $\begin{array}{l}\text { Amostra Geológica } \\
\quad(9 \text { analitos })\end{array}$ & $\begin{array}{l}\text { Calibração: } 25 \text { padrões certificados. } \\
\text { Modelos: seleção de variáveis e o } \\
\text { PLSR com dados centrados na média. }\end{array}$ & $\begin{array}{l}\text { Com exceção do teor do Ca, } \\
\text { modelo OLS é comparável } \\
\text { ao PLSR. }\end{array}$ & 52 \\
\hline
\end{tabular}


cont. Tabela 1

\begin{tabular}{|c|c|c|c|c|}
\hline Técnica & Matriz Amostral & Descrição & Resultados & Ref. \\
\hline NN & Polietileno dopado & $\begin{array}{l}\text { Sobreposição das linhas analíticas } \\
\text { dos dopantes (Re, Os, Ir e Pt). } \\
\text { Arquitetura otimizada da rede 100:4:4 }\end{array}$ & $\begin{array}{l}\text { Erro relativo: } 3,5 \% \\
\text { Tempo de treinamento: } \\
\text { 3-5 minutos }\end{array}$ & 16 \\
\hline NN, LR, CI & $\begin{array}{l}\text { Ligas } \\
\text { Fe-Ni-Cr }\end{array}$ & $\begin{array}{l}\text { Problema de interferência de matriz } \\
\text { Treinamento: } 100 \text { padrões } \\
\text { Arquitetura otimizada da rede } 12: 4: 3\end{array}$ & $\begin{array}{l}\text { NN: melhor exatidão } \\
\text { p/ Fe e Ni. CI: melhor } \\
\text { exatidão p/ } \mathrm{Cr}\end{array}$ & 38 \\
\hline $\begin{array}{c}\text { PCR, PLSR } \\
\text { e NN }\end{array}$ & $\mathrm{TiO}_{2}$ & $\begin{array}{l}\text { Controle de qualidade de } \mathrm{TiO}_{2} \\
\text { advindas de } 5 \text { métodos diferentes } \\
\text { de produção. Calibração: } 32 \text { amostras. }\end{array}$ & $\begin{array}{l}\text { NN: melhor precisão e } \\
\text { exatidão. PLSR e PCR: } \\
\text { apropriado p/ objetivo. }\end{array}$ & 37 \\
\hline $\begin{array}{l}\text { PLS, NN, } \\
\text { CI e LR }\end{array}$ & $\begin{array}{c}\mathrm{Pb} \text { e } \mathrm{S} \text { em } \\
\text { carvão ativado }\end{array}$ & $\begin{array}{l}\text { Problemas de interferência espectral. } \\
\text { Arquitetura da rede otimizada } 54: 7: 2 \\
\text { PLS: } 4 \text { componentes principais. }\end{array}$ & $\begin{array}{l}\text { NN: melhor modelo. } \\
\text { Desvio da previsão: } \\
\text { S } 4 \% \text { e } \mathrm{Pb} 9 \%\end{array}$ & 41 \\
\hline
\end{tabular}

CI: Coeficiente de Influência; PF: Parâmetros Fundamentais; PLSR: Regressão de Mínimos Quadrados Parciais; LR: Regressão Linear; MRL: Regressão Linear Múltipla; NN: Redes Neurais; PLS: PLSR e suas variantes.

\section{REFERÊNCIAS}

1. Christensen, L. H.; Drabaek, I.; Anal. Chim. Acta 1986, $188,15$.

2. Civici, N.; VanGrieken, R.; X-Ray Spectrom. 1997, 26, 147.

3. Henderson, C. M. B.; Cressey, G.; Redfern, S. A. T.; Radiat. Phys. Chem. 1995, 45, 459.

4. Mantler, M.; Schreiner, M.; X-Ray Spectrom. 2000, 29, 3.

5. Cesareo, R.; Gigante, G. E.; Castellano, A.; Rosales, M. A.; Aliphat, M.; DeLaFuente, F; Meitin, J. J.; Mendoza, A.; Iwanczyk, J. S.; Pantazis, J. A.; J. Trace Microprobe Tech. 1996, 14, 711 .

6. Török, S. B.; Lábár, J.; Schmeling, M.; Van Grieken, R. E.; Anal. Chem. 1998, 70, 495R.

7. Ellis, A. T.; Holmes, M.; Kregsamer, P.; Potts, P. J.; Streli, C.; West, M.; Wobrauschek, P.; J. Anal. At. Spectrom. 1998, 13, 209R.

8. Bradley, D. A.; Farquharson, M. J.; X-Ray Spectrom. 1999, 28, 270.

9. Zaichick, V.; Ovchjarenko, N.; Zaichick, S.; Appl. Radiat. Isot. 1999, 50, 283.

10. Leyden, D. E.; Fundamentals of X-Ray Spectrometry - as Applied to Energy Dispersive Techniques; Trator X-Ray, INC.; Califórnia, 1984; cap. 1, 2 e 7

11. Jenkins, R.; De Vries, J. L.; Practical X-Ray Spectrometry; $2^{\mathrm{a}}$ ed.; Springer-Verlag New York INC.; Hong Kong, 1970, cap. 01 .

12. Ewing, G. W.; Métodos Instrumentais de Análise Química; vol.01; Ed. Edgard Blucher; São Paulo, 1972; cap.09.

13. Leyden, D. E.; Spectroscopy 1987, 2, 28.

14. Jenkins, R.; X-Ray Spectrometry; vol.99; John Wiley \& Sons; Canadá, 1988, cap.07 e 09

15. Sahin, Y.; Karabulut, A.; Budak, G.; Appl. Spectrosc. Rev. 1996, 31, 333

16. Boger, Z.; Karpas, Z.; Anal. Chim. Acta 1994, 292, 243.

17. Bertin, E. P.; Principles and Practice of X-Ray Spectrometric Analysis; Plenum Press; Nova York, 1970, cap.01

18. Bosch Reig, F.; Gimeno Adelantado, J. V.; Peris Martínez, V.: Bosch Mossi, F.; Spectrochim. Acta 1995, 50B, 739.

19. Garivait, S.; Quisefit, J. P.; Chateaubourg, P.; Malingre, G.; X-Ray Spectrom. 1997, 26, 257.

20. García-González, M. T.; Haro-Ruiz, M. D.; HernándezLaguna, A.; Analyst 1992, 117, 1169.

21. Maenhaut, W.; Vandenhaute, J.; Duflou, H.; Fresenius Z. Anal. Chem. 1987, 326, 736.

22. Sherman, J.; Spectrochim. Acta 1955, 7, 283.

23. Rousseau, R. M.; Adv. X-Ray Anal. 1994, 37, 639.

24. Lachance, G. R.; Spectrochim. Acta 1993, 48B, 343.
25. Criss, J. W.; Birks, L. S.; Anal. Chem. 1968, 40, 1080.

26. de Vries, J. L.; Vrebos, B. A. R.; Handbook of X-Ray Spectrometry: Methods and Techniques; Van Grieken, R. E., Markowicz, A. A., Ed.; M. Dekker; Nova York, 1993; p.295.

27. Birks, L. S.; X-Ray Spectrochemical Analysis; vol.11; $2^{\mathrm{a}}$ ed.; Interscience Publishers; Nova York, 1969, cap.07 e 08.

28. Christensen, L. H.; Pind, N.; X-Ray Spectrom. 1981, 10, 156.

29. Sparks, C. J.; Adv. X-Ray Anal. 1976, 19, 19.

30. Martens, H.; Naes, T.; Multivariate Calibration; John Wiley \& Sons; Chichester, 1991, cap. 01 e 03.

31. Ferreira, M. M. C.; Antunes, A. M.; Melgo, M. S.; Volpe, P. L. O.; Quim. Nova 1999, 22, 724.

32. Wang, Y.; Zhao, X.; Kowalski, B. R.; Appl. Spectrosc. 1990, 44, 998.

33. Zupan, J.; Gasteiger, J.; Neural Networks for Chemists. An Introduction; VCH; Weinheim, 1993, cap. 01.

34. Bos, A.; Bos, M.; Van der Linden, W. E.;; Anal. Chim. Acta 1992, 256, 133.

35. Zupan, J.; Gasteiger, J.; Anal. Chim. Acta 1991, 248, 1.

36. Walczak, B.; Wegscheider, W.; Anal. Chim. Acta 1993, 283, 508.

37. Majcen, N.; Xavier Rius, F.; Zupan, J.; Anal. Chim. Acta 1997, 348, 87.

38. Bos, A.; Bos, M.; Van der Linden, W. E; Anal. Chim. Acta 1993, 277, 289.

39. Bos, M.; Weber, H. T.; Anal. Chim. Acta 1991, 247, 97.

40. Luo, L.; Guo, C.; Ma, G.; Ji, A.; X-Ray Spectrom. 1997, 26, 15.

41. Facchin, I.; Mello, C; Bueno, M. I. M. S.; Poppi, R. J.; X-Ray Spectrom. 1999, 28, 173.

42. Gunicheva, T. N.; Aisueva, T. S.; Afonin, V. P.; X-Ray Spectrom. 1995, 24, 187.

43. Hua, Y.; Yap, C. T.; Anal. Sci. 1994, 10, 867.

44. Finkel'shtein, A. L.; Gunicheva, T. N.; Afonin, V. P.; J. Anal. Chem. USSR 1984, 39, 306.

45. Wegrzynek, D; Holynska, B.; Ostachowicz, B.; Spectrochim. Acta 1998, 53B, 43.

46. Goldstein, S. J.; Slemmons, A. K.; Canavan, H. E.; Environ. Sci. Technol. 1996, 30, 2318.

47. Alvarez, M.; Mazo-Gray, V.; X-Ray Spectrom. 1991, 20, 67.

48. Marcó Parra, L. M.; Greaves, E. D.; Paz, J. L.; SajoBohus, L.; X-Ray Spectrom. 1993, 22, 362.

49. Honkimäki, V.; Hämäläinen, K.; Manninen S.; X-Ray Spectrom. 1996, 25, 215.

50. Swerts, J.; Van Espen, P.; Geladi, P.; Anal. Chem. 1993, 65, 1181.

51. Adams, M. J.; Allen, J. R.; J. Anal. Atom. Spectrom. 1998, 13, 119.

52. Adams, M. J.; Allen, J. R.; Analyst 1998, 123, 537. 